\title{
Redundancy Resolution of a Cartesian Space Operated Heavy Industrial Manipulator
}

\author{
M. Honegger, A. Codourey \\ Institute of Robotics, ETH-Zürich, CLA, 8092 Zürich, Switzerland \\ Tel. +41 163255 48, Fax +4116321087 \\ e-mail: honegger@ifr.mavt.ethz.ch
}

\begin{abstract}
In tunneling and mining construction work, heavy and large manipulators are used to spray liquid concrete on the walls. These manipulators are usually operated manually with simple units allowing to control all actuators independently. In cooperation between industry and university, a novel control system has been developed for the redundant heavy manipulator Robojet ${ }^{\circledR}$ that supports the operator by calculating the inverse kinematic model and controlling all actuators automatically. This paper presents the solution of the redundant inverse kinematics of the Robojet using the null-space method and its implementation in a control system.
\end{abstract}

\section{Introduction}

The MEYCO Robojet ${ }^{\circledR}$ sc-30 shown in figure 1 is a hydraulically actuated manipulator with application in tunneling construction work. It is used to spray liquid concrete on the walls of new tunnels. The design of this heavy and large manipulator with 8 degrees of freedom is ten years old, and the manipulator is being used worldwide. So far the manipulator has been operated manually with a simple control unit allowing to control the 8 actuators independently.

With this controller it is difficult to guide the jet along the wall of the tunnel while optimizing the spraying process and minimizing the losses of concrete. The operator must practice a long time in order to master the task in a satisfactory way.

We have developed a new control system that supports the operator in different ways. In one of the modes the operator can guide the jet directly in world-coordinates, using a space mouse, i.e. a 6 dof joystick [1]. In an automatic mode it is possible to scan the profile of the tunnel in a selected area using a laser scanner and to subsequently automatically control the distance and orientation between the jet and the wall. The operator needs only to guide the tool center point along the directions of the tunnel wall with the space mouse.

In both operation modes the movement given in cartesian coordinates has to be transformed in joint coordinates. The calculation of the redundant inverse kinematics and the control of the 8 hydraulic actuators is performed by the controller. The redundancies are solved either by using static conditions or by applying the well known nullspace method [2].

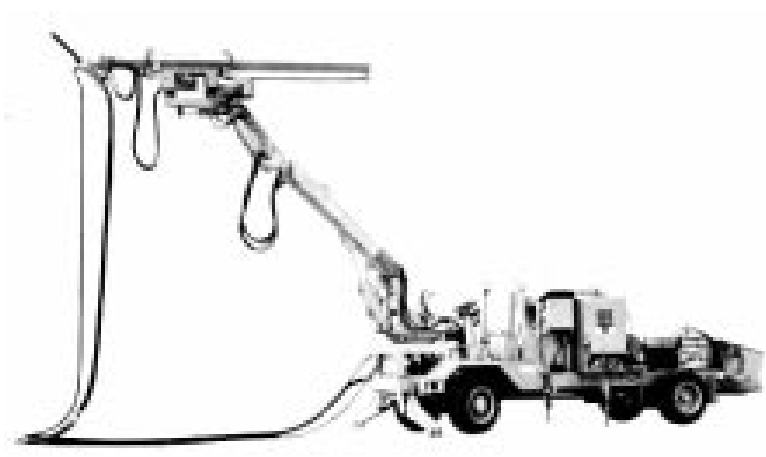

Fig.1: MEYCO Robojet sc-30

Chapter 2 and 3 present the kinematic model of the robot and its inverse solution. The following chapters describe its implementation in a simulation system and in the controller of the real robot.

\section{Kinematics}

The manipulator is mounted on a vehicle that is not moving during the spraying process. The location of the tool is therefore always described with reference to the vehicle.

Figure 2 shows a sketch of the kinematics of the robot with its redundant degrees of freedom. 


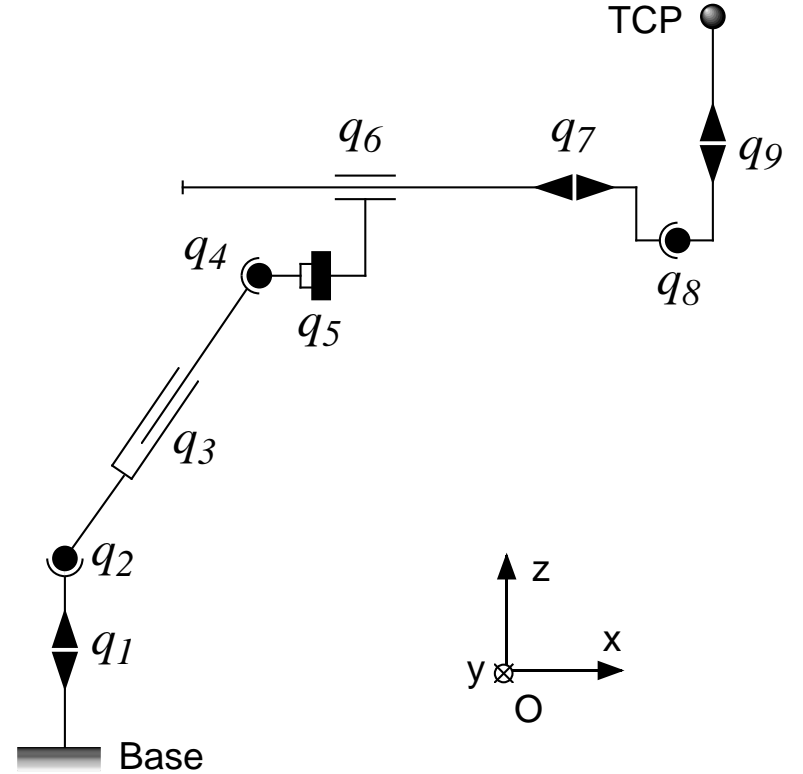

Fig.2: Kinematic model

The length of the manipulator when fully extended is bigger than $10 \mathrm{~m}$, thus allowing to spray in tunnels with a diameter of up to $25 \mathrm{~m}$ without moving the vehicle.

All 9 joints are hydraulically actuated. Joint 9 is used for a small circling motion of the jet for a better distribution of the sprayed concrete. It has no effect on the calculation of the kinematic model. The joint angles involved in the calculation are though

$$
q=\left[q_{1}, q_{2}, q_{3}, q_{4}, q_{5}, q_{6}, q_{7}, q_{8}\right]^{T} .
$$

The task requires the control of 5 dof of the jet, i.e. the position of the tool center point and 2 angles for the orientation. It is given by

$$
x=[x, y, z, \alpha, \beta]^{T} .
$$

The angle $\alpha$ describes the rotation about the $\mathrm{x}$-axis of the reference frame $\mathrm{O}$ leading to a new rotated frame $\mathrm{O}^{\prime} . \beta$ represents the rotation about the $y$-axis of the rotated frame O'. The calculation of the direct kinematic model $x=f(q)$ is straight forward using homogeneous transformation matrices. On the other hand calculating the inverse kinematics is not obvious. To solve the redundancies 3 constraints are required and no closed-form solution exists. A way to solve this problem is explained in the next section.

\section{Redundancy Resolution}

Many methods have been presented in the literature to solve this problem so far. In our case, two of them have been implemented and compared. The first one just uses some constraints for the position of the redundant axes. The second one is based on differential kinematics and uses the powerful null-space method.

\subsection{Constraints on the position}

As constraints for the redundant degrees of freedom, we used the following 3 static conditions for the variables:

$$
\begin{gathered}
q_{5}+\frac{q_{1}}{3}=0 \\
q_{4}-\frac{1+q_{2}}{6}=0 \\
q_{3}=\text { const }
\end{gathered}
$$

The selection of these equations can be understood as follows: In usual spraying tasks the position of the manipulator remains close to that of figure 2 . In this configuration the axes 1 and 5, and 2 and 4 respectively, have a similar orientation in space. This redundancy can be used to increase the workspace of the manipulator using equations (3) and (4). The consumption of oil of the 3rd joint during large translational motions is very high and can be moved slowly only. To simplify the control it is kept constant during the automated tasks (equation (5)). Its static position is given manually before executing the task.

For five given pose coordinates in the operational space and the three additional conditions, the angles of all eight joints are fully determined and can be calculated. Due to the complicated kinematic structure of the robot there is however no closed-form solution for the inverse kinematic model. The joint angles are thus calculated numerically with the Newton-Raphson method.

If this method guarantees a large workspace, it doesn't allow hight dynamics of operation because the velocity of the end-effector is limited by the slowest actuator. The null-space method is then used to solve this problem and explained in the following section.

\subsection{Null-space method}

Resolution of redundancy using the null-space method has already been presented many times in the literature. A good overview is given in [2] or [3]. It consists of resolving the redundancy by using the pseudoinverse of the Jacobian matrix of the manipulator for the movement of its TCP and the null-space of the Jacobian for internal movements. If $\dot{x}$ is the 5-dimensional velocity vector of 
the TCP and $q, \dot{q}$ the $n>5$ dimensional vector of joint angles and velocity respectively, then

$$
\dot{x}=J \dot{q} .
$$

To solve this equation with respect to $\dot{q}$, it is necessary to use its pseudoinverse, which yields the following result:

$$
\dot{q}=J^{\#} \dot{x}+\left(I-J^{\#} J\right) \dot{\phi}
$$

with

$$
J^{\#}=J^{T}\left(J J^{T}\right)^{-1}
$$

and where $\dot{\phi}$ is an arbitrary joint velocity vector and $\left(I-J^{\#} J\right) \dot{\phi}$ is its projection into the null-space of $J$. This corresponds actually to an internal motion of the manipulator which has no effect on the velocity of the end effector.

In order to optimize the motion of the manipulator with respect to some constraints, a weighted pseudoinverse can be used instead of the pseudoinverse. Equation (8) is then transformed to:

$$
J_{w}^{\#}=W^{-1} J^{T}\left(J W^{-1} J^{T}\right)^{-1}
$$

which corresponds to the minimization of the cost function $\dot{q}^{T} W \dot{q}[5]$.

Both of these techniques have been combined and applied to the Robojet so that optimization of the oil consumption and resolution of the redundancy can be achieved simultaneously. This is obtained by using the following equation:

$$
\dot{q}=J_{w}^{\#} \dot{x}+\left(I-J^{\#} J\right) \dot{\phi}
$$

\subsubsection{Selection of the weighting matrix}

In the case of the hydraulic manipulator Robojet, the objective is to minimize the oil consumption while moving. The oil consumption for each joint $i$ is proportional to the velocity of the hydraulic cylinder, i.e.

$$
Q_{i}=w_{i} \cdot\left|\dot{q}_{i}\right|
$$

Minimizing the oil consumption of the manipulator can be obtained by minimizing the following cost function:

$$
g(\dot{q})=\sum\left\|w_{i} \cdot \dot{q}_{i}\right\|^{2},
$$

or equivalently

$$
g(\dot{q})=\dot{q}^{T} \cdot W \cdot \dot{q},
$$

where $W=\operatorname{diag}\left(w_{1}^{2}, w_{2}^{2}, \ldots, w_{8}^{2}\right)$ is a diagonal matrix containing the squares of the consumption coefficients of each axis. The result of this optimization is actually given by equation (9).

However, in selecting the coefficients of the weighting matrix, we had to take into account that some joints can move quickly to their limits while others don't have enough oil to maintain the desired velocity. Thus, a fine parameter tuning and optimization was needed on the real manipulator. Our experiments have given the following matrix:

$$
W=\operatorname{diag}(37,78,100,39,25,25,12,9)
$$

A particular problem occured for joint 3. Compared to the other joints, the oil consumption of axis 3 is very high and thus the pump can not always deliver the needed amount of oil. This axis had to be weighted more than the others.

\subsubsection{Null-space motion}

As explained before, the use of the null-space method leads to internal motion of the manipulator without affecting the velocity of its end effector. The vector $\dot{\phi}$ can thus be used to perform a secondary task such as keeping the axes of the manipulator as close as possible to the middle of their workspace [4], avoiding obstacles [5] or other kind of tasks. In our case, the secondary objective is to keep some relations between the axes constant so as to optimize dynamically the workspace of the manipulator. The same static constraint equations as before ((3) and (4)) have been chosen for axes 1 and 2. Axis 3 on the other hand is now constrained by the position of axis 6 . The constraint equation is thus given by

$$
\frac{q_{6}}{3}-\frac{q_{3}}{1.85}=0 \text {. }
$$

Each of these constraints equations are used directly in the null-space by setting:

$$
\dot{\phi}=\left[\begin{array}{c}
k_{1}\left(q_{5}+\frac{q_{1}}{3}\right) \\
k_{2}\left(q_{4}-\frac{1+q_{2}}{6}\right) \\
k_{3}\left(\frac{q_{6}}{3}-\frac{q_{3}}{1.85}\right) \\
0 \\
0 \\
0 \\
0 \\
0
\end{array}\right]
$$


The internal velocity of each axis is thus adjusted until the constraints are satisfied. The coefficients $k_{i}, i=1 \ldots 3$ are used to tune the velocities of the internal motion.

\section{Simulation}

MOBILE, a simulation and 3D graphic animation software package [7] running on a SiliconGraphics workstation, is used to simulate the kinematics of the robot links and display its motions within a virtual tunnel (figure 3). The motions can be generated either by programming trajectories in a $\mathrm{C}++$ program or online using a space mouse to guide the jet in world coordinates.

This simulation has been used to test the null-space algorithms before their implementation in the real application and also for the first tuning of the weighting matrix W and the parameters $k_{i}$ for the internal motion.

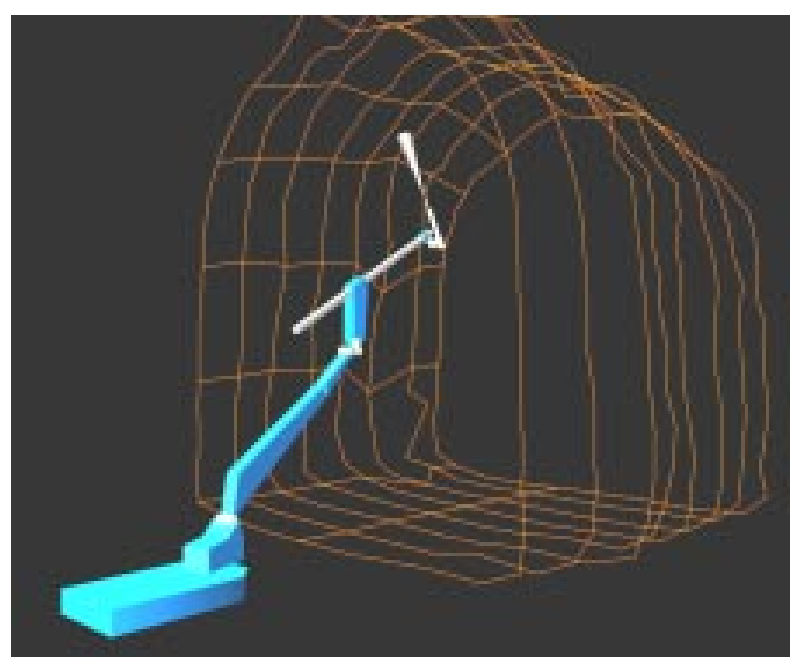

Fig.3: MOBILE simulation

As will be explained in the next section, the same space mouse is used for controlling the real robot. The 3D graphic animation can therefore also be used as a simulator for training purposes in a virtual reality environment.

\section{Implementation and Results}

In order to implement the new control system, the manipulator had to be equipped with encoders to measure the joint angles and with electrically controlled proportional valves for the hydraulic actuators. The sensors and actuators are connected to an Interbus-S peripheral bus system, that is controlled by a bus master board in a VMEbus chassis (see figure 4 ).

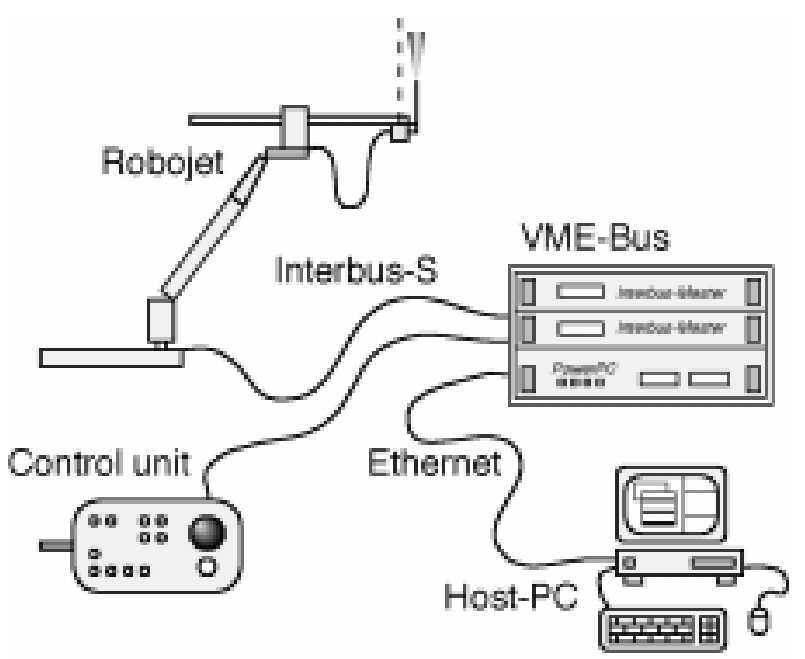

Fig.4: Hardware setup

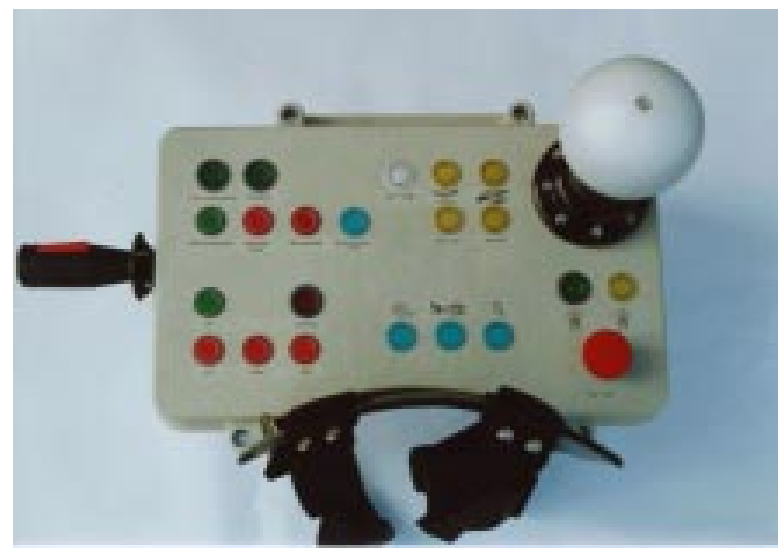

Fig.5: Control unit

The robot is operated by a control unit (figure 5) that allows to select different operating modes, to switch on the concrete pump, to start the measuring process and other functions. The chosen functions are confirmed by lighting of the corresponding buttons. The digital signals from the control unit are transfered to and from the VME-bus system via a second Interbus-S connection. Both the space mouse that is integrated in the control unit and the laser scanner, that is mounted on the manipulator near the jet and is needed to scan the tunnel profile for the automatic operation, are connected to the processor board of the VME-bus system with a RS232 serial line.

The control software including the calculation of the inverse kinematics is running on a Motorola PowerPC 603 processor board and is programmed in the object-oriented 
real-time system Denia/XOberon [8], [9]. A Host-PC allows the programming and monitoring of the system. It can further be used to change some process parameters that can't be controlled from the operator's control unit, such as the distance between the jet and the wall during automatic spraying, the number of measurements in the scanning process and others. It is also used to visualize these parameters and to display a protocol of the complete operation cycle.

After first tests of the new controller we have seen that the operation of the manipulator has become much easier than before. The training phase was reduced from about 1 week to only a couple of hours. Furthermore the quality of the spraying process could be increased. The operation of the robot in a real tunnel is shown in figure 6 .

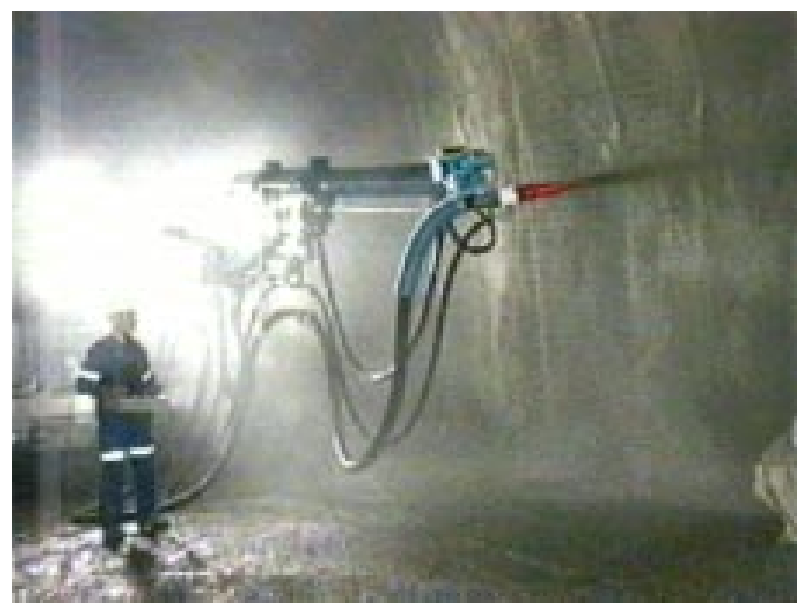

Fig.6: Robojet in operation

Compared to the method described in section 3.1, the use of the null-space method has the advantage of simultaneously optimizing the robots workspace and its dynamic performance.

\section{Conclusion}

The Robojet is a redundant heavy manipulator that used to be operated with simple manual control units by skilled personel.

In this paper, we presented a new control system that supports the operator in his work in different ways. The operator can guide the tool in cartesian coordinates directly using a space mouse. The calculation of the redundant inverse kinematics and the control of the actuators is performed by the control system. The null-space method is used to achieve a big workspace and a high dynamic performance simultaneously.
A first version of this control system has been implemented on a manipulator and first tests in real tunnels have been realized. With this controller the operation of the manipulator was greatly simplified. It reduces the costs of the training and increases the quality of work.

\section{Acknowledgments}

We would like to thank A. Hess and M. Trigo for their valuable help in the implementation of the null-space method as well as R. Hueppi and R. Brega for their support in the development of the controller. Further thanks to O. Tschumi of MEYCO equipment for the valuable cooperation.

This project has been partially supported by the Swiss Commission of Technology and Innovation.

\section{References}

[1] Space Control GmbH, 82205 Gilching Germany, website: http://www.op.dlr.de/FF-DR-RS/SC/

[2] Nakamura, Y., Advanced Robotics, Redundancy and Optimization, Addison-Wesley, 1991.

[3] Hollerbach, J.M., Suh, K.C., Redundancy Resolution of Manipulators through Torque Optimization, A.I. Memo 882, Massachusetts Institute of Technology, January 1986.

[4] Liegeois, A., Automatic supervisory control of the configuration and behavior of multibody mechanisms, IEEE Trans. Systems, Man, Cybern., SMC-7, pp. 868-871, 1977.

[5] Maciejewski, A. A., and Klein, C. A., Obstacle avoidance for kinematically redundant manipulators in dynamically varying environments, Int. J. Robotics Research, 4 no. 3, pp. 109-117, 1985.

[6] Whitney, D.E., The mathematics of coordinated control of prosthetic arms and manipulators, ASME Journal of Dynamic Systems, Meaurement and Control 94(4), pp. 303-309.

[7] A. Kecskemethy: MOBILE - Users guide and reference manual, IMECH GmbH, Duisburg/Moers Germany, 1994.

[8] D. Diez, S. Vestli: D'nia, an object-oriented real-time system, Real-Time Magazine, August 1995.

[9] ETH Inst.of Robotics, 8092 Zurich Switzerland, website: http://www.ifr.mavt.ethz.ch 